\title{
Subcutaneous or Transvenous Defibrillator Therapy
}

\author{
R.E. Knops, L.R.A. Olde Nordkamp, P.-P.H.M. Delnoy, L.V.A. Boersma, \\ J. Kuschyk, M.F. El-Chami, H. Bonnemeier, E.R. Behr, T.F. Brouwer, S. Kääb, \\ S. Mittal, A.-F.B.E. Quast, L. Smeding, W. van der Stuijt, A. de Weger, \\ K.C. de Wilde, N.R. Bijsterveld, S. Richter, M.A. Brouwer, J.R. de Groot, \\ K.M. Kooiman, P.D. Lambiase, P. Neuzil, K. Vernooy, M. Alings, T.R. Betts, \\ F.A.L.E. Bracke, M.C. Burke, J.S.S.G. de Jong, D.J. Wright, J.G.P. Tijssen, \\ and A.A.M. Wilde, for the PRAETORIAN Investigators"
}

ABSTRACT

The authors' full names, academic degrees, and affiliations are listed in the Appendix. Address reprint requests to Dr. Knops at the Department of Clinical and Experimental Cardiology, Amsterdam University Medical Centers, University of Amsterdam, Meibergdreef 9, 1105 AZ Amsterdam, the Netherlands, or at r.e.knops@amsterdamumc.nl.

*A full list of the PRAETORIAN investigators is provided in the Supplementary Appendix, available at NEJM.org.

N Engl J Med 2020;383:526-36. DOI: 10.1056/NEJMoa1915932

Copyright @ 2020 Massachusetts Medical Society.

\section{BACKGROUND}

The subcutaneous implantable cardioverter-defibrillator (ICD) was designed to avoid complications related to the transvenous ICD lead by using an entirely extrathoracic placement. Evidence comparing these systems has been based primarily on observational studies.

\section{METHODS}

We conducted a noninferiority trial in which patients with an indication for an ICD but no indication for pacing were assigned to receive a subcutaneous ICD or transvenous ICD. The primary end point was the composite of device-related complications and inappropriate shocks; the noninferiority margin for the upper boundary of the $95 \%$ confidence interval for the hazard ratio (subcutaneous ICD vs. transvenous ICD) was 1.45. A superiority analysis was prespecified if noninferiority was established. Secondary end points included death and appropriate shocks.

\section{RESULTS}

A total of 849 patients (426 in the subcutaneous ICD group and 423 in the transvenous ICD group) were included in the analyses. At a median follow-up of 49.1 months, a primary end-point event occurred in 68 patients in the subcutaneous ICD group and in 68 patients in the transvenous ICD group (48-month KaplanMeier estimated cumulative incidence, $15.1 \%$ and $15.7 \%$, respectively; hazard ratio, $0.99 ; 95 \%$ confidence interval $[\mathrm{CI}], 0.71$ to $1.39 ; \mathrm{P}=0.01$ for noninferiority; $\mathrm{P}=0.95$ for superiority). Device-related complications occurred in 31 patients in the subcutaneous ICD group and in 44 in the transvenous ICD group (hazard ratio, 0.69; 95\% CI, 0.44 to 1.09); inappropriate shocks occurred in 41 and 29 patients, respectively (hazard ratio, $1.43 ; 95 \% \mathrm{CI}, 0.89$ to 2.30 ). Death occurred in 83 patients in the subcutaneous ICD group and in 68 in the transvenous ICD group (hazard ratio, 1.23; $95 \% \mathrm{CI}, 0.89$ to 1.70); appropriate shocks occurred in 83 and 57 patients, respectively (hazard ratio, 1.52; 95\% CI, 1.08 to 2.12).

\section{CONCLUSIONS}

In patients with an indication for an ICD but no indication for pacing, the subcutaneous ICD was noninferior to the transvenous ICD with respect to devicerelated complications and inappropriate shocks. (Funded by Boston Scientific; PRAETORIAN ClinicalTrials.gov number, NCT01296022.) 
T MPLANTABLE CARDIOVERTER-DEFIBRILLAtors (ICDs) have been proven to be efficacious in the prevention of sudden cardiac death. ${ }^{1-3}$ Transvenous lead placement for cardiac sensing and defibrillation has been the standard for ICD design for several decades. However, important limitations of the technique include complications related to lead insertion, such as pneumothorax and cardiac perforation, and longterm complications, such as lead endocarditis and lead dysfunction. ${ }^{4}$ To avoid such complications, an entirely subcutaneous ICD was introduced as an alternative. ${ }^{5}$ The extrathoracic placement of the subcutaneous ICD circumvents the need to enter the heart and vasculature but makes it impossible for the device to deliver pacing therapy.

Class IIa recommendations for the subcutaneous ICD in U.S. and European guidelines for patients in whom pacing therapy for bradycardia, cardiac resynchronization, or antitachycardia pacing is not indicated are based on experience from observational studies. ${ }^{6-9}$ The Prospective Randomized Comparison of Subcutaneous and Transvenous Implantable Cardioverter Defibrillator Therapy (PRAETORIAN) trial evaluated whether the subcutaneous ICD would be noninferior to the transvenous ICD with regard to short-term and long-term device-related complications and inappropriate shocks.

\section{METHODS}

TRIAL DESIGN AND OVERSIGHT

We conducted this investigator-initiated, international, randomized, noninferiority trial in the United States and Europe. The principal coordinating investigators were responsible for the design of the trial, which has been published previously. ${ }^{10}$ The Academic Medical Center Amsterdam was responsible for site contracting, data collection, monitoring, and management. Trial design and execution were overseen by a steering committee, and the conduct of the trial and the safety of the patients were overseen by an independent data and safety monitoring board. The protocol, which is available with the full text of this article at NEJM.org, was approved by the institutional review board at all participating centers.

This trial was funded by Boston Scientific, which had no role in the design of the trial, analysis of the data, or the drafting and submission of the manuscript. The principal investigators analyzed the data in accordance with the statistical analysis plan and prepared the manuscript. The authors vouch for the accuracy and completeness of the data and for the fidelity of the trial to the protocol.

\section{TRIAL POPULATION}

Patients were eligible for entry into this trial if they were 18 years of age or older and had a class I or IIa indication for ICD therapy for primary or secondary prevention, according to the guidelines from the American College of Cardiology-American Heart Association Task Force on Clinical Practice Guidelines and the Heart Rhythm Society or those from the European Society of Cardiology. ${ }^{6,8}$ Key exclusion criteria were previous ICD implantation, unsuitability for subcutaneous ICD therapy according to QRS-Twave sensing analysis (see the Supplementary Appendix, available at NEJM.org), and indications for either bradycardia pacing or biventricular pacing. We also excluded patients with known ventricular tachycardia at a rate below 170 beats per minute or with refractory recurrent monomorphic ventricular tachycardia that could not be managed with medication or ablation therapy because, for such patients, antitachycardia pacing was considered to be an especially important therapeutic option. Detailed inclusion and exclusion criteria are provided in the Supplementary Appendix. All the patients provided written informed consent.

\section{RANDOMIZATION AND PROCEDURES}

Eligible patients were randomly assigned in a 1:1 ratio to receive either a subcutaneous ICD or transvenous ICD, with stratification according to center. Block sizes ranging from two to eight were used to conceal treatment assignments. All subcutaneous ICDs were manufactured by Cameron Health-Boston Scientific. The choice of transvenous ICD manufacturer was at the discretion of the physician performing the implantation. All transvenous ICDs were singlechamber devices unless a dual-chamber device was deemed to be necessary for the discrimination of arrhythmia. The procedures regarding implantation, defibrillation testing, and hospital discharge followed local clinical practice. All the patients were seen at a follow-up visit with- 
in 4 months after implantation. Thereafter, ICDs were interrogated at least twice per year, and patients had at least one annual visit to the outpatient clinic.

\section{ICD PROGRAMMING}

Programming of the parameters for the detection of ventricular tachycardia or ventricular fibrillation and for therapeutic variables was standardized and based on the best available evidence at the time of protocol development. ${ }^{11}$ Programming strategies were comparable between the two treatment groups. The cutoff for the fast ventricular tachycardia zone was set as close to 182 beats per minute as possible, given differences in manufacturer programming options, with one burst of antitachycardia pacing for the transvenous ICD. The cutoff for the ventricular fibrillation zone was 250 beats per minute. Deviation from the recommended device programming was allowed in order to fit the specific characteristics of the patient. Details are provided in the Supplementary Methods section in the Supplementary Appendix.

\section{END POINTS}

The composite primary end point of the trial consisted of device-related complications and inappropriate shocks. Complications included device infection that led to the extraction of the lead or generator; pocket hematoma that led to drainage, blood transfusion, or prolongation of hospitalization; device-related thrombotic events; pneumothorax or hemothorax that led to intervention or prolongation of hospitalization; cardiac perforation or tamponade; lead repositioning or replacement; and other complications related to the lead or generator that led to medical or surgical intervention. An ICD shock was classified as inappropriate when it was delivered for any rhythm other than ventricular fibrillation or ventricular tachycardia. Secondary end points included the individual components of the primary end point, death from any cause, appropriate ICD therapy (including antitachycardia pacing), major adverse cardiac events, hospitalization for heart failure, and crossover between the assigned devices.

A clinical-events committee consisting of three electrophysiologists who were not otherwise involved in the trial adjudicated all clinical and arrhythmic events. Shock therapy and other ar- rhythmic events that were derived from regular device interrogation were classified as being either appropriate or inappropriate according to the rhythm that initiated the therapy. Hence, shocks without electrograms were not adjudicated. A complete overview of trial end points and definitions is provided in the Supplementary Appendix.

\section{STATISTICAL ANALYSIS}

The trial was designed to test the hypothesis of noninferiority of the subcutaneous ICD as compared with the transvenous ICD with respect to the time from device implantation to the first occurrence of a primary end-point event. The noninferiority margin for the upper boundary of the $95 \%$ confidence interval for the hazard ratio was set at 1.45. The estimation of cumulative incidence and sample-size justification have been reported previously. ${ }^{10}$ We estimated that the occurrence of a primary end-point event in the transvenous ICD group would be $17.2 \%$ at 48 months. ${ }^{1,11}$ Assuming that $5 \%$ of patients would discontinue the trial, we calculated that the enrollment of 425 patients in each group would provide the trial with $85 \%$ power to show noninferiority of the subcutaneous ICD at a one-sided alpha level of 0.025 . A superiority analysis was prespecified if noninferiority was established.

Analyses for all the end points were performed in the modified intention-to-treat population, which included patients according to the group to which they had been randomly assigned, regardless of the device they received. Patients who did not receive either device after randomization or who underwent randomization in error were excluded from the analyses. A sensitivity analysis was performed in the astreated population, which included patients according to the treatment that they first received. Additional sensitivity analyses included, among others, a competing-risks analysis to account for death and incomplete follow-up, an analysis to account for missing electrographic data from the device, and a multiple imputation analysis by fully conditional specification. ${ }^{12}$

For the time-to-event analyses, cumulative incidence curves were constructed with the use of the Kaplan-Meier method, and hazard ratios and $95 \%$ confidence intervals were calculated by Cox proportional-hazards models. For these analyses, missing data were presumed to be missing 


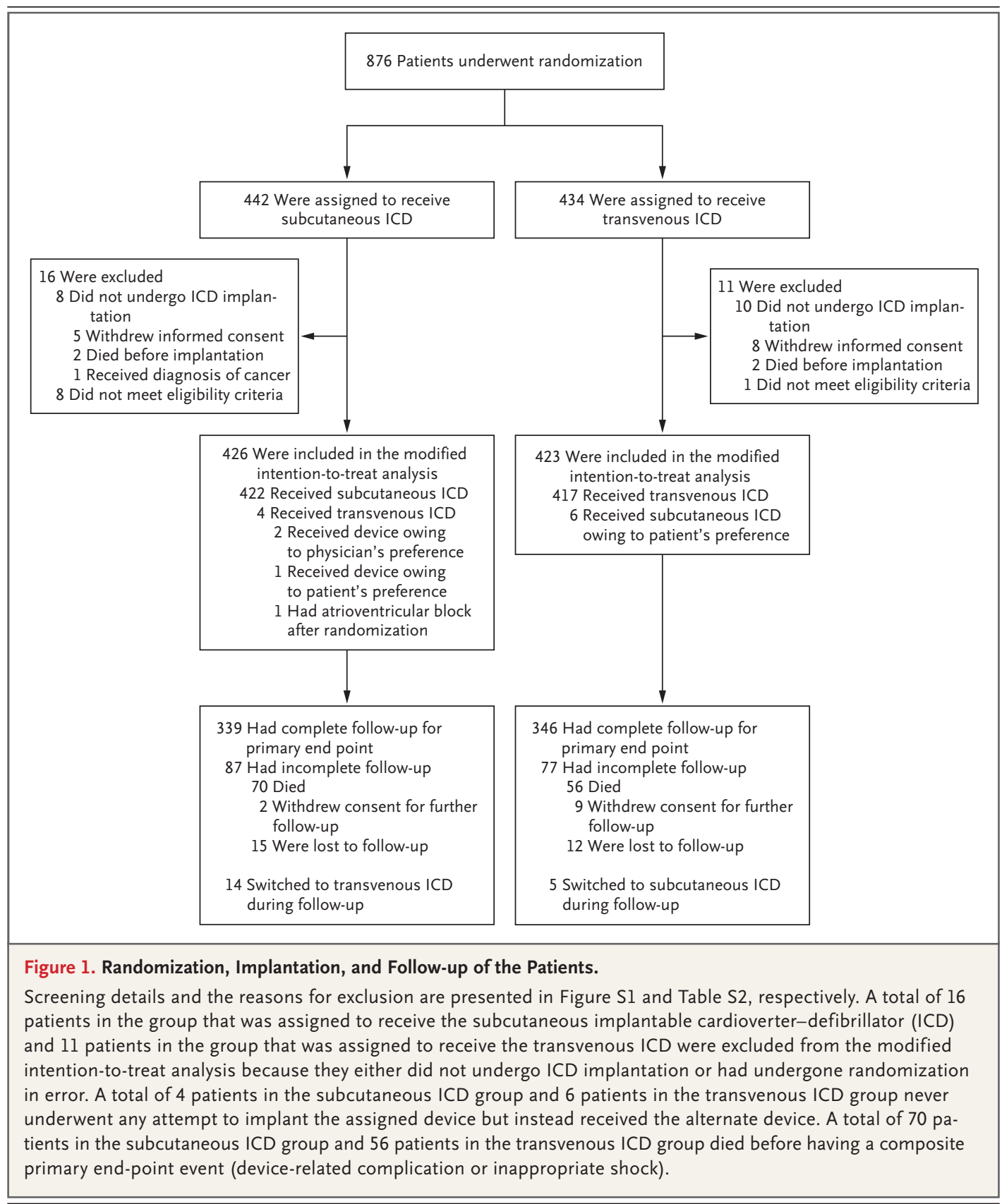

at random, and data were censored for patients with incomplete follow-up on the last known event-free day. The confidence intervals were not adjusted for multiplicity and therefore should not be used to infer definitive treatment effects. Prespecified subgroups that were defined according to age, sex, and body-mass index were analyzed for the occurrence of a primary endpoint event. Additional information regarding the statistical analyses is provided in the Supple- mentary Appendix. Statistical analyses were performed with the use of $\mathrm{R}$ software, version 3.6.2 ( $R$ Core Team).

\section{RESULTS}

\section{PATIENTS AND IMPLANTATIONS}

From March 2011 through January 2017, a total of 876 patients were enrolled at 39 centers in Europe and the United States (Fig. 1 and Table S1 


\begin{tabular}{|c|c|c|}
\hline Characteristic & $\begin{array}{c}\text { Subcutaneous ICD } \\
(N=426)\end{array}$ & $\begin{array}{c}\text { Transvenous ICD } \\
(\mathrm{N}=423)\end{array}$ \\
\hline Median age (IQR) - yr & $63(54-69)$ & $64(56-70)$ \\
\hline Female sex - no. (\%) & $89(20.9)$ & $78(18.4)$ \\
\hline \multicolumn{3}{|l|}{ Diagnosis — no. (\%) } \\
\hline Ischemic cardiomyopathy & $289(67.8)$ & $298(70.4)$ \\
\hline Nonischemic cardiomyopathy & $99(23.2)$ & $98(23.2)$ \\
\hline Genetic arrhythmia syndrome & $20(4.7)$ & $18(4.3)$ \\
\hline Hypertrophic cardiomyopathy & $15(3.5)$ & $7(1.7)$ \\
\hline Idiopathic ventricular fibrillation & $11(2.6)$ & $5(1.2)$ \\
\hline Congenital heart disease & $3(0.7)$ & $3(0.7)$ \\
\hline Other $\uparrow$ & $4(0.9)$ & $1(0.2)$ \\
\hline Secondary prevention — no. (\%) & $80(18.8)$ & $84(19.9)$ \\
\hline Median ejection fraction (IQR) — \% & $30(25-35)$ & $30(25-35)$ \\
\hline Mean QRS duration - msec & $105 \pm 19$ & $105 \pm 20$ \\
\hline \multicolumn{3}{|l|}{ NYHA class — no./total no. (\%) } \\
\hline 1 & $144 / 423(34.0)$ & $134 / 421(31.8)$ \\
\hline II & $205 / 423(48.5)$ & $223 / 421(53.0)$ \\
\hline III or IV & $74 / 423(17.5)$ & $64 / 421(15.2)$ \\
\hline Median body-mass index (IQR) & $27.0(24.5-30.5)$ & $27.9(25.2-31.7)$ \\
\hline Hypertension or use of antihypertensive drugs — no./total no. (\%) & $227 / 424(53.5)$ & $240 / 419(57.3)$ \\
\hline Hypercholesterolemia or use of lipid-lowering drugs - no./total no. (\%) & $161 / 419(38.4)$ & $175 / 418$ (41.9) \\
\hline Current or recent smoking — no./total no. (\%) & $119 / 406(29.3)$ & $139 / 401(34.7)$ \\
\hline Diabetes mellitus — no./total no. (\%) & $112 / 426(26.3)$ & $126 / 421(29.9)$ \\
\hline Previous CABG — no./total no. (\%) & $86 / 425(20.2)$ & $85 / 421(20.2)$ \\
\hline History of atrial fibrillation — no./total no. (\%) & $115 / 426(27.0)$ & $93 / 420(22.1)$ \\
\hline History of nonsustained ventricular tachycardia — no./total no. (\%) & $46 / 423(10.9)$ & $44 / 417(10.6)$ \\
\hline History of syncope — no./total no. (\%) & $23 / 420(5.5)$ & $33 / 418(7.9)$ \\
\hline \multicolumn{3}{|l|}{ Site location — no. (\%) } \\
\hline Europe & $394(92.5)$ & $395(93.4)$ \\
\hline United States & $32(7.5)$ & $28(6.6)$ \\
\hline Median time from randomization to device implantation (IQR) — days & $7.5(1.0-29.0)$ & $6.0(1.0-26.5)$ \\
\hline \multicolumn{3}{|c|}{$\begin{array}{l}\text { Plus-minus values are means } \pm \text { SD. CABG denotes coronary-artery bypass grafting, ICD implantable cardioverter-defi- } \\
\text { brillator, IQR interquartile range, and NYHA New York Heart Association. } \\
\text { The patients in this category had ventricular fibrillation due to coronary spasm (one patient in the subcutaneous ICD } \\
\text { group and one in the transvenous ICD group), coronary dissection (one in the subcutaneous ICD group), ischemic stroke } \\
\text { (one in the subcutaneous ICD group), and myocarditis (one in the subcutaneous ICD group). } \\
\text { The body-mass index is the weight in kilograms divided by the square of the height in meters. }\end{array}$} \\
\hline
\end{tabular}

in the Supplementary Appendix). Information about patients who underwent screening and reasons for exclusion are provided in Figure S1 and Table S2. Of the 876 patients enrolled, 27 were excluded from the primary analysis; $18 \mathrm{did}$ not undergo ICD implantation and 9 had undergone randomization in error. A total of $849 \mathrm{pa}-$ tients were included in the primary analysis; of these patients, 426 were randomly assigned to the subcutaneous ICD group and 423 to the transvenous ICD group (Fig. 1). The clinical characteristics of the patients at baseline were similar in the two groups (Table 1). The median age of the patients was 63 years (interquartile 
range, 55 to 70 ); $19.7 \%$ of the patients were women, and $69.1 \%$ had ischemic cardiomyopathy. The median left ventricular ejection fraction was $30 \%$.

Details of the initial implantation procedure are provided in Table S3. Of the patients in the transvenous ICD group, 48 (11.3\%) received a dual-chamber device at initial implantation. A total of 10 patients crossed over to the other device without any attempt to implant the assigned device. Five additional patients crossed over shortly after initial implantation, and 14 patients crossed over during follow-up. Details of all the crossovers are provided in Table S4. Details of adherence to the device programming protocol are provided in Table S5.

\section{FOLLOW-UP AND PRIMARY END POINT}

Follow-up of the trial was completed on December 1, 2019. A total of 339 patients in the subcutaneous ICD group and 346 patients in the transvenous ICD group had complete follow-up (Fig. 1). The median duration of follow-up was 49.1 months (48.0 months in the subcutaneous ICD group and 50.6 months in the transvenous ICD group). The primary end point occurred in 68 patients in the subcutaneous ICD group and in 68 patients in the transvenous ICD group (48-month Kaplan-Meier estimated cumulative incidence, $15.1 \%$ and $15.7 \%$, respectively). The hazard ratio for the primary end point was 0.99 (95\% confidence interval [CI], 0.71 to 1.39 ; noninferiority margin, $1.45 ; \mathrm{P}=0.01$ for noninferiority; $\mathrm{P}=0.95$ for superiority) (Fig. 2A and Table 2).

Device-related complications occurred in 31 patients in the subcutaneous ICD group and in 44 patients in the transvenous ICD group (cumulative incidence, $5.9 \%$ and $9.8 \%$, respectively; hazard ratio, 0.69 ; $95 \%$ CI, 0.44 to 1.09 ) (Fig. 2B). The incidence of complications within the first 30 days was $3.8 \%$ in the subcutaneous ICD group and $4.7 \%$ in the transvenous ICD group. The incidence of complications related to the ICD lead was lower in the subcutaneous ICD group than in the transvenous ICD group (1.4\% vs. 6.6\%) (Fig. S2).

Inappropriate shocks occurred in 41 patients in the subcutaneous ICD group and in 29 patients in the transvenous ICD group (cumulative incidence, $9.7 \%$ and $7.3 \%$, respectively; hazard ratio, 1.43; 95\% CI, 0.89 to 2.30) (Fig. 2C). The first occurrences of inappropriate shocks in the subcutaneous ICD group were most frequently caused by cardiac oversensing (in $58.5 \%$ of the patients with an inappropriate shock), whereas inappropriate shocks in the transvenous ICD group were more commonly triggered by supraventricular arrhythmia (in 93.1\%) (Table 2). Deviations from the programming protocol at the time of first inappropriate shocks are described in the Supplementary Results section in the Supplementary Appendix; the findings indicated that two episodes of inappropriate shocks in the transvenous ICD group may have been prevented if the prespecified programming had been used.

The findings of the primary analysis were consistent in the as-treated population (Fig. S3). There were no between-group differences in the occurrence of the primary end point across prespecified subgroups (Fig. S4). Results of the competing-risks analyses, multivariable analyses, and sensitivity analyses that account for missing data are provided in Tables S6 through S11.

\section{SECONDARY END POINTS}

During the course of the trial, 83 patients in the subcutaneous ICD group and 68 patients in the transvenous ICD group died (hazard ratio, 1.23; 95\% CI, 0.89 to 1.70) (Table 3 and Fig. S5). Causes of death are presented in Table S12. In each group, 18 patients died suddenly.

Appropriate ICD shocks were more frequent in patients in the subcutaneous ICD group than in those in the transvenous ICD group $(19.2 \%$ vs. $11.5 \%$; hazard ratio, 1.52; 95\% CI, 1.08 to 2.12 ) (Table 3 and Fig. S6) and included subcutaneous ICD shocks due to oversensing of ventricular tachycardia below the programmed therapy zone in 11 patients. Appropriate antitachycardia pacing was delivered in $12.9 \%$ of the patients in the transvenous ICD group and successfully terminated $55 \%$ of all treated episodes of ventricular tachycardia. A total of 5 patients in the subcutaneous ICD group underwent implantation of a transvenous device for pacing for the treatment of bradycardia. There were no between-group differences in the cumulative incidence of major adverse cardiac events, hospitalization for heart failure, or total crossovers (Table 3), although there were numerically more crossovers during follow-up (shortly after the implantation attempt or later in follow-up) from the subcutaneous ICD to the transvenous ICD (14 patients) than vice versa (5 patients). 


\section{A Primary Composite End Point}

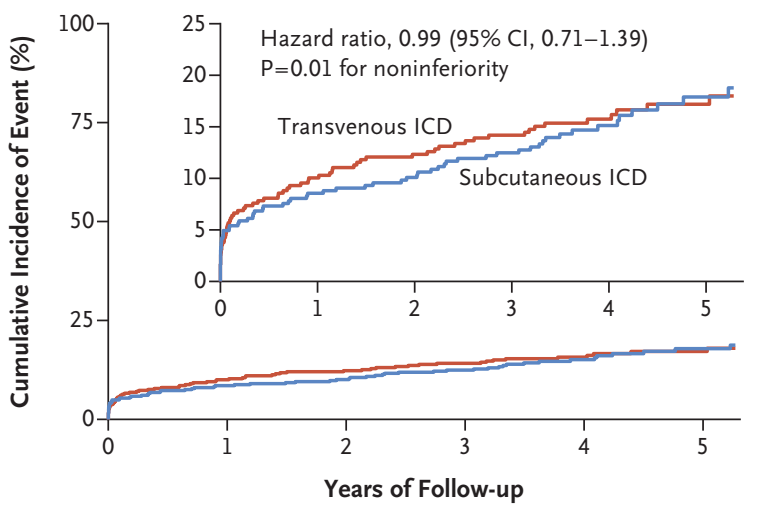

No. at Risk

$\begin{array}{lllllll}\text { Transvenous ICD } & 423 & 359 & 338 & 313 & 192 & 105\end{array}$ $\begin{array}{lllllll}\text { Subcutaneous ICD } & 426 & 366 & 342 & 317 & 182 & 108\end{array}$

B Device-Related Complications

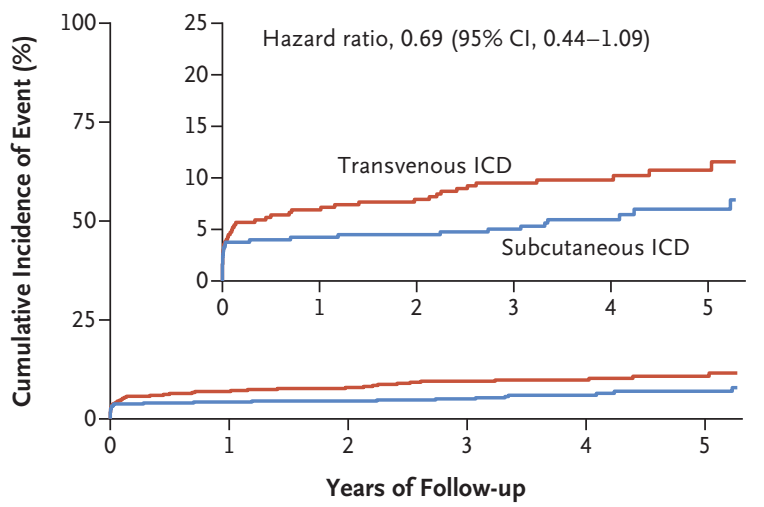

No. at Risk

$\begin{array}{lllllll}\text { Transvenous ICD } & 423 & 372 & 355 & 331 & 210 & 112\end{array}$

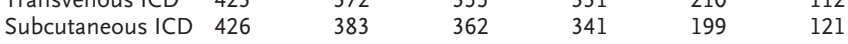

C Inappropriate Shocks

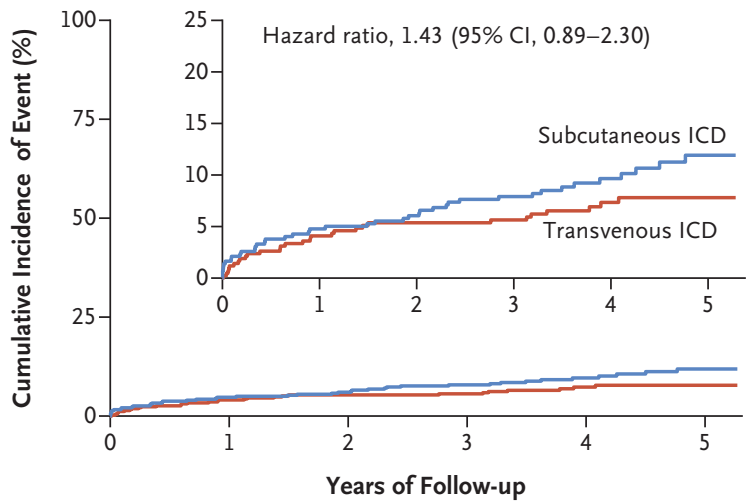

No. at Risk

$\begin{array}{lllllll}\text { Transvenous ICD } & 423 & 383 & 363 & 340 & 210 & 119\end{array}$

$\begin{array}{lllllll}\text { Subcutaneous ICD } & 426 & 382 & 358 & 333 & 198 & 117\end{array}$
Figure 2. Time-to-First-Event Curves for the Primary End Point and Its Components.

Shown is the cumulative incidence of the first occurrence of the composite primary end point (Panel $A$ ) and its components, the first device-related complication (Panel B) and the first inappropriate shock (Panel C). Hazard ratios were derived from Cox regressions and indicate the relative risk (subcutaneous ICD vs. transvenous ICD) of the end point. The $95 \%$ confidence intervals were not adjusted for multiple comparisons and therefore should not be used to infer definitive treatment effects. Insets show the same data on an enlarged y axis.

\section{DISCUSSION}

In this trial, we found that the subcutaneous ICD was noninferior to the transvenous ICD with respect to device-related complications or inappropriate shocks in patients with an indication for defibrillator therapy but with no indication for pacing. The results were consistent in several sensitivity analyses and subgroup analyses. We observed equal numbers of sudden cardiac deaths in the two groups, but there were numerically more deaths from other causes in the subcutaneous ICD group than in the transvenous ICD group.

With respect to the two components of the primary outcome, there was a higher cumulative incidence of device-related complications in the transvenous ICD group and a higher cumulative incidence of inappropriate shocks in the subcutaneous ICD group, although the trial was not powered for these comparisons. Perspectives may vary among physicians and patients about which component poses a heavier burden: whereas complications are associated primarily with physical distress, ICD shocks can have profound psychological implications. ${ }^{13}$

The overall incidence of complications in this trial was as anticipated and was similar to that in previous studies. ${ }^{4,14,15}$ Fewer lead-related complications (including infection, perforation, lead dislodgement, and lead dysfunction) and subsequent surgical reinterventions occurred in the subcutaneous ICD group than in the transvenous ICD group, but this effect was counterbalanced by more frequent pocket hematomas with the subcutaneous ICD. The use of general anesthesia and defibrillation testing was much greater with the subcutaneous ICD than with the 


\begin{tabular}{|c|c|c|c|}
\hline End point & $\begin{array}{l}\text { Subcutaneous ICD } \\
\quad(N=426)\end{array}$ & $\begin{array}{l}\text { Transvenous ICD } \\
(\mathrm{N}=423)\end{array}$ & $\begin{array}{l}\text { Hazard Ratio } \\
\text { (95\% CI) }\end{array}$ \\
\hline Primary composite end point — no. (\%) & $68(15.1)$ & $68(15.7)$ & $0.99(0.71-1.39) \dagger$ \\
\hline \multicolumn{4}{|l|}{ Components of primary end point } \\
\hline Device-related complication — no. (\%) & $31(5.9)$ & $44(9.8)$ & $0.69(0.44-1.09)$ \\
\hline Infection - no.t & 4 & 8 & \\
\hline Bleeding - no. & 8 & 2 & \\
\hline Thrombotic event - no. & 1 & 2 & \\
\hline Pneumothorax - no. $\delta$ & 0 & 4 & \\
\hline Lead perforation - no. $\delta$ & 0 & 4 & \\
\hline Tamponade - no. & 0 & 2 & \\
\hline Lead repositioning - no. $\mathbb{\int}$ & 2 & 7 & \\
\hline Other lead or device complication — no. & 19 & 20 & \\
\hline Lead replacement \\
& 3 & 9 & \\
\hline Device malfunction & 4 & 6 & \\
\hline Sensing issues & 4 & 0 & \\
\hline Pacing indication $\|$ & 5 & 1 & \\
\hline Implantation failure & 0 & 3 & \\
\hline Defibrillation test failure $* *$ & 3 & 0 & \\
\hline Pain or discomfort & 2 & 3 & \\
\hline Inappropriate shock - no. (\%)† & $41(9.7)$ & $29(7.3)$ & $1.43(0.89-2.30)$ \\
\hline $\begin{array}{l}\text { Atrial fibrillation or supraventricular tachycardia } \\
\qquad- \text { no. }\end{array}$ & 11 & 27 & \\
\hline Cardiac oversensing - no.tr & 24 & 2 & \\
\hline Noncardiac oversensing - no. $\iint$ & 8 & 0 & \\
\hline
\end{tabular}

* Percentages are 4-year cumulative incidences based on Kaplan-Meier estimates in time-to-first-event analyses. Multiple end points could occur in one patient; only the first end point was included in the estimation of the cumulative incidence. For all end points, the sample included all the patients in the trial group. The widths of the $95 \%$ confidence intervals have not been adjusted for multiplicity and therefore should not be used to infer definitive treatment effects.

$\dagger \mathrm{P}=0.01$ for noninferiority; $\mathrm{P}=0.95$ for superiority.

$\mp$ This category included lead-related infections in one patient in the subcutaneous ICD group and in five in the transvenous ICD group.

$\int$ This end point was included in the composite end point "lead-related complications" (Fig. S2).

If In the subcutaneous ICD group, lead replacements were due to dislocation in two patients and to myopotential oversensing in one. In the transvenous ICD group, lead replacements were due to lead dysfunction in six patients and to lead dislodgement in three.

\| In the subcutaneous ICD group, three patients received a pacemaker, one received a cardiac-resynchronization therapy device with a defibrillator (CRT-D), and one crossed over to transvenous ICD therapy - all for pacing for the treatment of bradycardia. In the patient in the transvenous ICD group who had previously crossed over to subcutaneous ICD therapy, sick-sinus syndrome later developed, for which a pacemaker was implanted.

$* *$ This category included defibrillator test failures that led to surgical reintervention.

†T The subcutaneous ICD sensing filter (SMART Pass) was not activated or was unavailable in $78 \%$ of the first inappropriate shocks in the subcutaneous ICD group.

trt This category included T-wave and P-wave oversensing and includes shock on atrial fibrillation or supraventricular tachycardia below the detection limit in five patients in the subcutaneous ICD group.

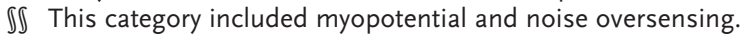

transvenous ICD. Longer-term follow-up of this cohort will be important because the incidence of lead-related complications increases over time with the transvenous ICD ${ }^{16}$ and because battery longevity is a limiting factor for the subcutaneous ICD. ${ }^{17}$ 


\begin{tabular}{|c|c|c|c|}
\hline End Point & $\begin{array}{c}\text { Subcutaneous ICD } \\
(N=426)\end{array}$ & $\begin{array}{c}\text { Transvenous ICD } \\
(\mathrm{N}=423)\end{array}$ & $\begin{array}{c}\text { Hazard Ratio } \\
(95 \% \mathrm{Cl})\end{array}$ \\
\hline Death from any cause - no. (\%) & $83(16.4)$ & $68(13.1)$ & $1.23(0.89-1.70)$ \\
\hline Sudden cardiac death - no. $†$ & 18 & 18 & \\
\hline Death from other cardiovascular causes - no. & 34 & 28 & \\
\hline Death from noncardiovascular causes - no. & 31 & 22 & \\
\hline Appropriate shock therapy — no. (\%) & $83(19.2)$ & $57(11.5)$ & $1.52(1.08-2.12)$ \\
\hline Ventricular fibrillation - no. & 32 & 22 & \\
\hline Ventricular tachycardia within therapy zone - no. & 57 & 41 & \\
\hline Ventricular tachycardia below therapy zone - no. $\downarrow$ & 11 & 0 & \\
\hline \multicolumn{4}{|l|}{ Antitachycardia pacing - no. (\%)』 } \\
\hline Appropriate & $6(0.6)$ & $54(12.9)$ & \\
\hline Inappropriate & $1(0.3)$ & $30(7.2)$ & \\
\hline Major adverse cardiac event — no. (\%) & $64(13.3)$ & $80(16.4)$ & $0.80(0.57-1.11)$ \\
\hline Hospitalization for heart failure — no. (\%) & $79(17.4)$ & $74(16.1)$ & $1.08(0.79-1.49)$ \\
\hline Crossover to other study device — no. (\%) & $18(4.3)$ & $11(2.7)$ & $1.64(0.77-3.47)$ \\
\hline Before initial implantation - no. & 4 & 6 & \\
\hline During implantation or follow-up - no. & 14 & 5 & \\
\hline Upgrade to CRT-D — no. (\%) & $16(3.5)$ & $21(4.2)$ & \\
\hline \multicolumn{4}{|c|}{$\begin{array}{l}\text { * Percentages are 4-year cumulative incidences based on Kaplan-Meier estimates in time-to-first-event analyses. For all } \\
\text { end points, the sample included all the patients in the trial group. The widths of the } 95 \% \text { confidence intervals were not } \\
\text { adjusted for multiplicity and therefore should not be used to infer definitive treatment effects. } \\
\text { This category included death from unexplained causes. } \\
+ \text { These shocks were delivered on ventricular tachycardia below the programmed therapy limit with oversensing of car- } \\
\text { diac signals. The sensing filter (SMART Pass) was not activated or was unavailable in } 91 \% \text { of the first occurrences of } \\
\text { such events. } \\
\text { 『 Patients who received antitachycardia pacing in the subcutaneous ICD group had previously crossed over to transve- } \\
\text { nous ICD therapy or had received a CRT-D. }\end{array}$} \\
\hline
\end{tabular}

Patients with a subcutaneous ICD had a higher risk of inappropriate shock than anticipated; the shocks were most frequently precipitated by oversensing of cardiac signals (predominantly T waves) and noncardiac signals. Although shocks on supraventricular arrhythmias can generally be managed with device reprogramming or medication, shocks caused by cardiac or noncardiac oversensing are less modifiable. However, a sensing filter that attenuates oversensing, which resulted in a $50 \%$ reduction of first inappropriate shocks in an earlier study, was introduced in a later stage of the trial. ${ }^{18}$ This filter was unavailable or not activated in the majority (78\%) of patients with a subcutaneous ICD during their first inappropriate shock. Therefore, this trial could not assess the potential benefit of the sensing filter. Such changes to device technology may improve the future performance of the subcutaneous ICD.

We observed a higher cumulative incidence of appropriate shocks with the subcutaneous ICD than with the transvenous ICD, which was, for the most part, explained by the inability of the subcutaneous ICD to deliver antitachycardia pacing. Antitachycardia pacing terminated ventricular tachycardia in more than half the pacing attempts with the transvenous ICD. In addition, the sensing of the subcutaneous ICD, which is based on morphologic features, can result in double-counting of slow ventricular tachycardia occurring at a rate below the programmed therapy zone, thus causing the sensed rate (if both QRS complexes and $\mathrm{T}$ waves are counted) to exceed the therapy threshold. According to the end-point definition, which was based on earlier 
ICD trials, these shocks were classified as appropriate but - although occasionally clinically desirable - could be considered to be unnecessary. ${ }^{19,20}$

This trial has several limitations. First, the members of the clinical-events committee were aware of the trial-group assignments. Second, device technology evolved throughout the trial, and practitioners who performed the implantations had less experience with the subcutaneous ICD than with the transvenous ICD, which could have affected clinical outcomes. Third, screening data were incomplete, and thus selection bias could not be ruled out. Fourth, 27 patients were excluded before device implantation, 38 patients were lost to follow-up, and 126 patients died before having a primary end-point event. However, sensitivity analyses yielded consistent results. Fifth, it is debatable whether the magnitude of the possible between-group difference as reflected by the noninferiority margin is clinically acceptable. Finally, the median follow-up of 48 months was too limited to provide information on chronic complications. Long-term followup is therefore warranted and is ongoing.

The results of our trial showed that among patients with an indication for ICD therapy but not for pacing therapy, the subcutaneous ICD was noninferior to the transvenous ICD with respect to the cumulative incidence of the primary end point of device-related complications or inappropriate shocks.

A data sharing statement provided by the authors is available with the full text of this article at NEJM.org.

Supported by Boston Scientific.

Dr. Knops reports receiving consulting fees from Boston Scientific, Abbott, and Medtronic; Dr. Delnoy, receiving grant support from Abbott, Boston Scientific, Medtronic, Biotronik, MicroPort, and Cook Medical; Dr. Boersma, receiving grant support, paid to his institution, from Boston Scientific, serving as a primary investigator of the UNTOUCHED S-ICD (Understanding Outcomes with the EMBLEM S-ICD in Primary Prevention Patients with Low EF Study), and serving on a steering committee for the EMPOWER trial, the PRAETORIAN DFT (Prospective Randomized Comparative Trial of Subcutaneous Implantable Cardioverter-Defibrillator Implantation with and without Defibrillation Testing) trial, the ASE (Acute Feasibility Investigation of a New S-ICD Electrode Arrangement for Reduction of Defibrillation Energies) trial, and the EFFORTLESS S-ICD (Evaluation of Factors Impacting Clinical Outcome and Cost Effectiveness of the S-ICD) trial; Dr. Kuschyk, receiving consulting fees and lecture fees from Boston Scientific; Dr. El-Chami, receiving consulting fees from Boston Scientific and Medtronic; Dr. Bonnemeier, receiving grant support from Boston Scientific, Medtronic, and Sorin Group; Dr. Behr, receiving grant support from Boston Scientific; Dr. Mittal, receiving consulting fees and advisory board fees from Boston Scientific; Dr. Richter, receiving grant support, paid to Heart Center Leipzig-Leipzig Heart Institute, from Boston Scientific and grant support, paid to his institution, from Abbott and Biotronik; Dr. de Groot, receiving grant support from Abbott, AtriCure, and Boston Scientific, grant support and lecture fees from Medtronic, Bayer, and Daiichi Sankyo, consulting fees from Atrian Medical, Novartis, and Servier, and lecture fees from Johnson \& Johnson; Ms. Kooiman, receiving consulting fees from Boston Scientific and Biotronik; Dr. Lambiase, receiving grant support and lecture fees from Boston Scientific, Abbott, and Medtronic; Dr. Vernooy, receiving grant support, paid to the Cardiovascular Research Institute Maastricht, from Medtronic, Abbott, and Biotronik; Dr. Alings, receiving consulting fees from Bayer, Boehringer Ingelheim, Bristol-Myers Squibb, Daiichi Sankyo, Pfizer, Sanofi, and Portola Pharmaceuticals; Dr. Betts, receiving lecture fees from Boston Scientific; Dr. Burke, receiving grant support, consulting fees, and lecture fees from Boston Scientific and consulting fees from and equity in AtaCor Medical; and Dr. Wright, receiving grant support from Boston Scientific and consulting fees and lecture fees from Medtronic. No other potential conflict of interest relevant to this article was reported.

Disclosure forms provided by the authors are available with the full text of this article at NEJM.org.

We thank the patients who participated in this trial; Aeilko H. Zwinderman, Ph.D., for assistance in performing the statistical analyses; the members of the clinical-events committee for adjudicating all events; and the members of the data and safety monitoring board for contributions to the conduct of this trial.

\section{APPENDIX}

The authors' full names and academic degrees are as follows: Reinoud E. Knops, M.D., Ph.D., Louise R.A. Olde Nordkamp, M.D., Ph.D., Peter-Paul H.M. Delnoy, M.D., Ph.D., Lucas V.A. Boersma, M.D., Ph.D., Jürgen Kuschyk, M.D., Mikhael F. El-Chami, M.D., Hendrik Bonnemeier, M.D., Ph.D., Elijah R. Behr, M.D., Tom F. Brouwer, M.D., Ph.D., Stefan Kääb, M.D., Ph.D., Suneet Mittal, M.D., Anne-Floor B.E. Quast, M.D., Ph.D., Lonneke Smeding, Ph.D., Willeke van der Stuijt, M.D., Anouk de Weger, M.Sc., Koen C. de Wilde, M.D., Nick R. Bijsterveld, M.D., Ph.D., Sergio Richter, M.D., Marc A. Brouwer, M.D., Ph.D., Joris R. de Groot, M.D., Ph.D., Kirsten M. Kooiman, M.P.A., Pier D. Lambiase, M.D., Ph.D., Petr Neuzil, M.D., Ph.D., Kevin Vernooy, M.D., Ph.D., Marco Alings, M.D., Ph.D., Tim R. Betts, M.D., Ph.D., Frank A.L.E. Bracke, M.D., Ph.D., Martin C. Burke, D.O., Jonas S.S.G. de Jong, M.D., Ph.D., David J. Wright, M.D., Jan G.P. Tijssen, Ph.D., and Arthur A.M. Wilde, M.D., Ph.D.

The authors' affiliations are as follows: the Heart Center, Department of Clinical and Experimental Cardiology, Amsterdam Cardiovascular Sciences, Amsterdam University Medical Centers, University of Amsterdam (R.E.K., L.R.A.O.N., L.V.A.B., T.F.B., A.-F.B.E.Q., L.S., W.S., A.W., K.C.W., J.R.G., K.M.K., M.C.B., J.G.P.T., A.A.M.W.), ERN GUARD-Heart (E.R.B., P.D.L., A.A.M.W.), and the Department of Cardiology, OLVG (J.S.S.G.J.), Amsterdam, the Department of Cardiology, Isala Heart Centre, Zwolle (P.-P.H.M.D.), the Department of Cardiology, St. Antonius Hospital, Nieuwegein (L.V.A.B.), the Department of Cardiology, Flevoziekenhuis, Almere (N.R.B.), the Department of Cardiology, Radboud University Medical Center, Nijmegen (M.A.B.), the Department of Cardiology, Cardiovascular Research Institute Maastricht, Maastricht University Medical Center, Maastricht (K.V.), the Department of Cardiology, Amphia Hospital, Breda (M.A.), Werkgroep Cardiologische Centra Nederland, Utrecht (M.A.), and the Department of Electrophysiology, Catharina Hospital, Eindhoven (F.A.L.E.B.) - all in the Netherlands; the First Department of Medicine-Cardiology, University Medical Center 
Mannheim, and the German Center for Cardiovascular Research Partner Site Heidelberg-Mannheim, Mannheim (J.K.), Klinik für Innere Medizin III, Schwerpunkt Kardiologie und Angiologie, Universitätsklinikum Schleswig-Holstein, Campus Kiel, Kiel (H.B.), the Department of Medicine I, Ludwig-Maximilians University Hospital, and the German Center for Cardiovascular Research, Munich Heart Alliance, Munich (S.K.), and the Department of Electrophysiology, Heart Center at University of Leipzig, Leipzig (S.R.) — all in Germany; the Division of Cardiology Section of Electrophysiology, Emory University, Atlanta (M.F.E.-C.); the Cardiology Clinical Academic Group, St. George's, University of London and St. George's University Hospitals NHS Foundation Trust London (E.R.B.), and Office of the Director of Clinical Electrophysiology Research and Lead for Inherited Arrhythmia Specialist Services, University College London and Barts Heart Centre (P.D.L.), London, the Oxford Biomedical Research Centre, Oxford University Hospitals NHS Trust, Oxford (T.R.B.), and Liverpool Heart and Chest Hospital, Liverpool (D.J.W.) - all in the United Kingdom; Valley Health System, Ridgewood, NJ (S.M.); the Department of Cardiology, Homolka Hospital, Prague, Czech Republic (P.N.); and CorVita Science Foundation, Chicago (M.C.B.).

REFERENCES

1. Bardy GH, Lee KL, Mark DB, et al. Amiodarone or an implantable cardioverter-defibrillator for congestive heart failure. N Engl J Med 2005;352:225-37.

2. Moss AJ, Hall WJ, Cannom DS, et al. Improved survival with an implanted defibrillator in patients with coronary disease at high risk for ventricular arrhythmia. N Engl J Med 1996;335:1933-40.

3. Moss AJ, Zareba W, Hall WJ, et al. Prophylactic implantation of a defibrillator in patients with myocardial infarction and reduced ejection fraction. $\mathrm{N}$ Engl J Med 2002;346:877-83.

4. Kirkfeldt RE, Johansen JB, Nohr EA, Jorgensen OD, Nielsen JC. Complications after cardiac implantable electronic device implantations: an analysis of a complete, nationwide cohort in Denmark. Eur Heart J 2014;35:1186-94.

5. Bardy GH, Smith WM, Hood MA, et al. An entirely subcutaneous implantable cardioverter-defibrillator. N Engl J Med 2010;363:36-44.

6. Al-Khatib SM, Stevenson WG, Ackerman MJ, et al. 2017 AHA/ACC/HRS guideline for management of patients with ventricular arrhythmias and the prevention of sudden cardiac death: executive summary: a report of the American College of Cardiology/American Heart Association Task Force on Clinical Practice Guidelines and the Heart Rhythm Society. Circulation 2018;138(13):e210-e271.

7. Burke MC, Gold MR, Knight BP, et al. Safety and efficacy of the totally subcutaneous implantable defibrillator: 2-year results from a pooled analysis of the IDE Study and EFFORTLESS Registry. J Am Coll Cardiol 2015;65:1605-15.
8. Priori SG, Blomström-Lundqvist C, Mazzanti A, et al. 2015 ESC guidelines for the management of patients with ventricular arrhythmias and the prevention of sudden cardiac death: the Task Force for the Management of Patients with Ventricular Arrhythmias and the Prevention of Sudden Cardiac Death of the European Society of Cardiology (ESC) endorsed by: Association for European Paediatric and Congenital Cardiology (AEPC). Europace 2015;17:1601-87.

9. Weiss R, Knight BP, Gold MR, et al. Safety and efficacy of a totally subcutaneous implantable-cardioverter defibrillator. Circulation 2013;128:944-53.

10. Olde Nordkamp LRA, Knops RE, Bardy $\mathrm{GH}$, et al. Rationale and design of the PRAETORIAN trial: a Prospective, RAndomizEd comparison of subcuTaneOus and tRansvenous ImplANtable cardioverter-defibrillator therapy. Am Heart J 2012;163(5):753.e2-760.e2.

11. Wilkoff BL, Williamson BD, Stern RS, et al. Strategic programming of detection and therapy parameters in implantable cardioverter-defibrillators reduces shocks in primary prevention patients: results from the PREPARE (Primary Prevention Parameters Evaluation) study. J Am Coll Cardiol 2008;52:541-50.

12. Liu Y, De A. Multiple imputation by fully conditional specification for dealing with missing data in a large epidemiologic study. Int J Stat Med Res 2015;4:287-95.

13. Sears SF, Rosman L, Sasaki S, et al. Defibrillator shocks and their effect on objective and subjective patient outcomes: results of the PainFree SST clinical trial. Heart Rhythm 2018;15:734-40.
14. Boersma LV, El-Chami MF, Bongiorni MG, et al. Understanding outcomes with the EMBLEM S-ICD in primary prevention patients with low EF study (UNTOUCHED): clinical characteristics and perioperative results. Heart Rhythm 2019;16:163644.

15. Brouwer TF, Yilmaz D, Lindeboom R, et al. Long-term clinical outcomes of subcutaneous versus transvenous implantable defibrillator therapy. J Am Coll Cardiol 2016;68:2047-55.

16. Koneru JN, Jones PW, Hammill EF, Wold N, Ellenbogen KA. Risk factors and temporal trends of complications associated with transvenous implantable cardiac defibrillator leads. J Am Heart Assoc 2018;7(10):e007691.

17. Quast A-FBE, van Dijk VF, Yap S-C, et al. Six-year follow-up of the initial Dutch subcutaneous implantable cardioverterdefibrillator cohort: Long-term complications, replacements, and battery longevity. J Cardiovasc Electrophysiol 2018;29: 1010-6.

18. Theuns DAMJ, Brouwer TF, Jones PW, et al. Prospective blinded evaluation of a novel sensing methodology designed to reduce inappropriate shocks by the subcutaneous implantable cardioverter-defibrillator. Heart Rhythm 2018;15:1515-22.

19. Moss AJ, Schuger C, Beck CA, et al. Reduction in inappropriate therapy and mortality through ICD programming. N Engl J Med 2012;367:2275-83.

20. Poole JE, Johnson GW, Hellkamp AS, et al. Prognostic importance of defibrillator shocks in patients with heart failure. N Engl J Med 2008;359:1009-17.

Copyright (C) 2020 Massachusetts Medical Society. 